\title{
Distinct Regions of the Periaqueductal Gray Are Involved in the Acquisition and Expression of Defensive Responses
}

\author{
Beatrice M. De Oca, Joseph P. DeCola, Stephen Maren, and Michael S. Fanselow \\ Department of Psychology, University of California, Los Angeles, Los Angeles, California 90024-1563
}

In fear conditioning, a rat is placed in a distinct environment and delivered footshock. The response to the footshock itself is called an activity burst and includes running, jumping, and vocalization. The fear conditioned to the distinct environment by the footshock elicits complete immobility termed freezing. Lesions of the ventral periaqueductal gray (vPAG) strongly attenuate freezing. However, lesions of the dorsolateral periaqueductal gray (dIPAG) increase the amount of freezing seen to conditional fear cues acquired under conditions in which intact rats do not demonstrate much fear conditioning. To examine the necessity of these regions in the acquisition and expression of fear, we performed five experiments that examined the effects of electrolytic lesions of the dIPAG and the VPAG in learned and unlearned fear. In experiment 1, lesions of the vPAG strongly attenuated, whereas lesions of the dIPAG en- hanced, unconditional freezing to a cat. In experiment 2, lesions of the dIPAG made before but not after training enhanced the amount of freezing shown to conditional fear cues acquired via immediate footshock delivery. In experiment 3, vPAG lesions made either before or after training with footshock decreased the level of freezing to conditional fear cues. Neither dIPAG lesions nor vPAG lesions affected footshock sensitivity (experiment 4) or consumption on a conditioned taste aversion test that does not elicit antipredator responses (experiment 5). On the basis of these results, it is proposed that activation of the dIPAG produces inhibition of the VPAG and forebrain structures involved with defense. In contrast, the VPAG seems to be necessary for postencounter freezing defensive behavior.

Key words: periaqueductal gray; midbrain; freezing; fear conditioning; unconditional fear; defense
When rats are placed in a distinct environment and shocked, two behaviors can be observed. Circastrike responding involves running, jumping, and vocalization and is elicited directly by footshock. The other response is freezing. Freezing is complete immobility except for that required for breathing. Freezing is not elicited directly by the shock but by the fear to the chamber conditioned by the footshock (Blanchard and Blanchard, 1969; Bolles and Collier, 1976; Fanselow, 1980). Electrolytic lesions of the dorsolateral periaqueductal gray (dlPAG) result in enhanced freezing to contextual cues paired with footshock under circumstances in which intact rats freeze little (Fanselow et al., 1995).

Because dIPAG lesions facilitate conditional fear, three roles for the dlPAG are possible: (1) the dlPAG may inhibit the amygdala or other forebrain structures involved in processing fearprovoking stimuli; (2) the dlPAG may interfere with the performance of fear-motivated behavior; and (3) the dIPAG may produce a brief analgesia elicited by shocks that in turn interferes with the acquisition of fear.

One technique in which support for the second option has been obtained is fear-potentiated startle. In fear-potentiated startle, the acoustic startle response is enhanced by cues signaling footshock. There is a nonmonotonic function between the shock intensity used during fear conditioning and the potentiated startle. When a conditional stimulus (CS) is trained with high shock intensities, there is a diminution of the magnitude of the subsequent potenti-

\footnotetext{
Received May 27, 1997; revised Feb. 9, 1998; accepted Feb. 13, 1998.

This work was supported by National Institute of Mental Health Grant MH 39786 to M.S.F.

Correspondence should be addressed to Dr. Beatrice M. De Oca, Department of Social Sciences, Western New Mexico University, Box 680, 1000 West College Avenue, Silver City, NM 88062.

Dr. Maren's present address: Department of Psychology, University of Michigan, 525 East University Avenue, Ann Arbor, MI 48109-1109.

Copyright (ㄷ) 1998 Society for Neuroscience $0270-6474 / 98 / 183426-07 \$ 05.00 / 0$
}

ation of startle compared with intermediate shock intensities (Davis and Astrachan, 1978). Because potentiated startle levels increased during the course of extinction of conditional fear, an inverse relationship between fear and potentiated startle is suggested. Lesions of the dIPAG made after fear conditioning blocked the attenuation of potentiated startle seen with high shock intensities, allowing for the levels of fear potentiation that occur with high shock intensities and with intermediate shock intensities to be similar. In addition, stimulation of the dIPAG with nontoxic doses of kainic acid attenuated the potentiated startle elicited in the presence of cues fear-conditioned with moderate shock (Walker and Davis, 1997). Thus, stimulation of the dlPAG during fearpotentiated startle to a cue trained with moderate levels of shock produced behavior typical of that seen when high shock intensities were used. The authors concluded that the dIPAG may inhibit amygdaloid terminals that synapse onto the fear-potentiated startle circuit at the level of nucleus reticularis pontis caudalis.

An instance of shock interfering with acquisition instead of performance occurs with massed and immediate shock delivery. When shocks are delivered close together in time (Fanselow and Tighe, 1988) or immediately after the placement of a rat in the chamber (Blanchard and Blanchard, 1971), no conditional fear is acquired. One reason may be that shock interferes with the processing of the context by the animal (Fanselow, 1986), thus compromising the context-shock association. That dIPAG lesions attenuate these effects (Fanselow et al., 1995) suggests that activation of the dIPAG in intact rats may interfere with or inhibit other structures like the amygdala that are involved in the acquisition of conditional fear. However, when the shocks are widely spaced or delivered after contextual cues are processed, conditioning can still occur, suggesting a brief rather than a sustained inhibition by the dIPAG. If the lesion indeed prevents the inhibition of other struc- 
tures during conditioning, then only lesions made before testing but not after should eliminate the effect of massed or immediate shock delivery. This hypothesis will be tested in experiment 2 .

The model proposed by Fanselow (1991) suggests that the ventral periaqueductal gray (vPAG) is exclusively involved in the expression of fear, whereas forebrain structures such as the amygdala that communicate with the PAG are involved in acquisition. In contrast to this view, there are data suggesting a role for the vPAG in the acquisition of conditional fear. Administration of acoustic startle stimuli produces both startle and freezing. Presumably, this freezing occurs because the apparatus serves as a CS and the startle cues serve as an unconditional stimulus (US). Conditional fear to apparatus cues and habituation of acoustic startle are negatively correlated (Leaton and Borszcz, 1985). Procedures that weaken fear conditioning like latent inhibition or extinction of the CS facilitate habituation of startle responses. Lesions of the vPAG also facilitated habituation of acoustic startle (Borszcz et al., 1989). According to the authors, fear conditioned to the apparatus serves to sensitize responses to the acoustic stimuli, and lesions of the vPAG attenuated this conditional fear. However, when behavioral and autonomic measures of conditional fear are assessed, only the behavioral response freezing was attenuated by lesions of the PAG (LeDoux et al., 1988). Another goal of the present research is to see whether the vPAG plays a critical role exclusively during training.

The present series of experiments examines the behavior of rats with electrolytic dIPAG and vPAG lesions in response to both unlearned and learned danger stimuli to determine the role of these structures in the acquisition and expression of defensive behavior.

\section{MATERIALS AND METHODS}

Subjects, surgery, and histology. The subjects were 126 adult Long-Evansderived rats born and maintained at the University of California, Los Angeles, Psychology Department vivarium. The animals were $\sim 120 \mathrm{~d}$ old at the start of the experiment. Rats were individually housed in standard hanging stainless steel cages, fed food and water ad libitum, and maintained on a 14:10 hr light/dark cycle (lights on at 7:00 A.M.). All procedures were conducted during the light portion of the cycle. Rats were handled daily for 2-3 d before surgery. In the fourth and fifth experiments, the same 30 adult male Long-Evans-derived rats from experiment 1 served as subjects. These rats received either dIPAG, $\mathrm{vPAG}$, or sham lesions.

Rats were anesthetized with sodium pentobarbital $(55 \mathrm{mg} / \mathrm{kg}$, i.p.), treated with atropine sulfate $(0.12 \mathrm{mg} / \mathrm{kg})$, and placed in a stereotaxic device with the head in a level position. A single incision was made on the scalp, the skull was exposed, and a small hole was made in the skull with a dental drill. Electrolytic lesions were made in the dIPAG (stereotaxic coordinates from bregma: anteroposterior, $-7.3 \mathrm{~mm},-7.8 \mathrm{~mm}$; lateral, + $0.7 \mathrm{~mm}$; and dorsoventral, $-5.6 \mathrm{~mm}$ ) or vPAG (stereotaxic coordinates from bregma: anteroposterior, $-7.5 \mathrm{~mm},-7.8 \mathrm{~mm}$; lateral, $+0.7 \mathrm{~mm}$; and dorsoventral, $-6.0 \mathrm{~mm}$ ) with a monopolar electrode. The electrode consisted of a stainless steel insect pin insulated with Epoxylite except for 250 $\mu \mathrm{m}$ at the blunt tip. Lesions were made by passing DC current (Grass; D. C. Constant Current Lesion Maker, model D. C. LM5A) for $10 \mathrm{sec}(0.7$ and $0.6 \mathrm{~mA}$ for dIPAG and vPAG, respectively). The electrode was not lowered for sham lesions. After surgery, the rats were allowed to recover on a heating pad before being returned to their home cages. All subjects received $7 \mathrm{~d}$ of recovery from surgery during which time they were handled daily. At the conclusion of the experiments, rats received an overdose of sodium pentobarbital and were transcardially perfused with $0.9 \%$ saline followed by $10 \%$ formalin. Brains were removed and fixed in formalin before being sectioned ( $50 \mu \mathrm{m}$ coronal sections) on a cryostat. Every third section was mounted on a glass slide and subsequently stained with thionin. Lesion locations were verified using a dissecting scope and reconstructed on standard atlas templates.

Lesions are preferred over direct chemical stimulation because they allow the naturally occurring responses of the animal to danger stimuli to be observed. Furthermore, lesions allow one to infer whether the le- sioned area is necessary for the production of defensive behavior. Electrolytic lesions will be used instead of excitotoxic lesions because of the risks associated with infusion of neurotoxins in close proximity to the cerebral aqueduct. Furthermore, all previous parametric work with these procedures has used electrolytic lesions. The continued use of electrolytic lesions will allow direct comparison between the behavioral results with these experiments and other experiments done in the laboratory without confounding lesion type.

Behavioral apparatus. The testing apparatus of the first experiment consisted of a large $60 \times 58.5 \times 61 \mathrm{~cm}$ plywood container with a clear acrylic ceiling and a clear acrylic glass door through which all behavior was observed and video recorded. Inside the enclosure, proximal to the door in one corner, was an empty, clear glass aquarium $(24.5 \times 25.5 \times$ $25.5 \mathrm{~cm}$ ) with a hinged door on the top; the rat was placed in this enclosure for the test. The ceiling of the aquarium had a small protruding spout, allowing for air to enter and exit the aquarium. On sessions in which the cat was used, it was placed inside the larger enclosure such that the rat and cat could see and smell each other, but contact between the two was impossible. The cat was an adult domestic cat.

The apparatus used in all other experiments consisted of an observation chamber $(21 \times 28 \times 20.5 \mathrm{~cm})$ made of stainless steel walls and a clear acrylic ceiling and door. The chamber contained a stainless steel rod floor, with $180.4 \mathrm{~mm}$ rods placed $1.0 \mathrm{~cm}$ apart, center to center. Before each use, the chambers were cleaned with a $5 \%$ ammonium hydroxide solution, and a light coating of this ammonium hydroxide solution was placed in the litter pans underneath the grid floor. The chamber lay within a soundattenuating chest that was free of its door to facilitate observation by the experimenter. Scrambled electric shock originated from a custom $450 \mathrm{~V} \mathrm{AC}$ shock source wired through a mechanical scrambler (Lafayette Instruments Co.). This equipment was in an isolated room of the laboratory that was lit by two fluorescent ceiling fixtures.

Behavioral testing procedures. In the first experiment, male rats received either dlPAG, vPAG, or sham lesions. Each rat was individually placed into the glass aquarium for a $3 \mathrm{~min}$ baseline observation period. Twentyfour hours later, each rat was again placed inside the aquarium for the cat exposure test. The cat remained inside the larger enclosure for the duration of the test session, whereas each rat was individually placed inside the aquarium and observed for $512 \mathrm{sec}$. Freezing was operationally defined as complete immobility except for that necessitated by breathing in all experiments and was scored using a time-sampling procedure; every $2 \mathrm{sec}$, the rat was determined to be freezing or not freezing by an experimenter who was blind to the lesion condition of the rat.

In all of the fear-conditioning experiments, conditional fear was assessed by placement in the conditioning chambers for $512 \mathrm{sec}$. Each rat was time-sampled every $8 \mathrm{sec}$. No shocks were presented during this test.

In experiment 2 , female rats with either dIPAG or sham lesions were placed in a conditioning chamber and immediately administered a single footshock ( $1 \mathrm{sec} ; 1 \mathrm{~mA})$ or not shocked. The shock was delivered as soon as the door to the chamber was shut, $\sim 2 \mathrm{sec}$ after placement of the rats in the chamber. All rats remained in the chamber for $64 \mathrm{sec}$. This procedure was repeated for $3 \mathrm{~d}$. Rats remained undisturbed in their home cages for $2 \mathrm{~d}$ after this training procedure. On the third day after training, half of the rats that received sham lesions on the first surgery received lesions of the dIPAG, and all other rats received sham lesions. Thus, rats received either a dIPAG lesion before or after training or sham lesions. After the second surgery, there was a 1 week recovery period before testing during which they were handled daily.

In the third experiment, female rats received either sham or vPAG lesions. After recovery from surgery, rats were placed in conditioning chambers for $3 \mathrm{~min}$. After the $3 \mathrm{~min}$, half of the rats were administered three $(1 \mathrm{sec} ; 1 \mathrm{~mA})$ shocks, spaced $64 \mathrm{sec}$ apart, and half received no shock but merely remained in the chamber for the same duration as the shocked rats. Rats remained undisturbed in their home cages for $2 \mathrm{~d}$ after fear conditioning. On the third day, half of the rats that received sham lesions on the first surgery received lesions of the vPAG; all other rats received sham lesions. Thus, rats received either a vPAG lesion before or after training or sham lesions.

In experiment 4, a VARIAC transformer connected to a shock scrambler (LaFayette Instruments Co.) was used to deliver shocks to the floor of the chamber. Shocks $1 \mathrm{sec}$ in duration were delivered in steps of 0.066 $\mathrm{mA}$; the shocks were delivered in an ascending series and were separated by $10 \mathrm{sec}$. The intensity at which each rat flinched, jumped, and vocalized was observed by one experimenter, who was blind to the lesion condition of each rat, and recorded by a second experimenter, who controlled the shock delivery. Once vocalization was elicited, the procedure was re- 

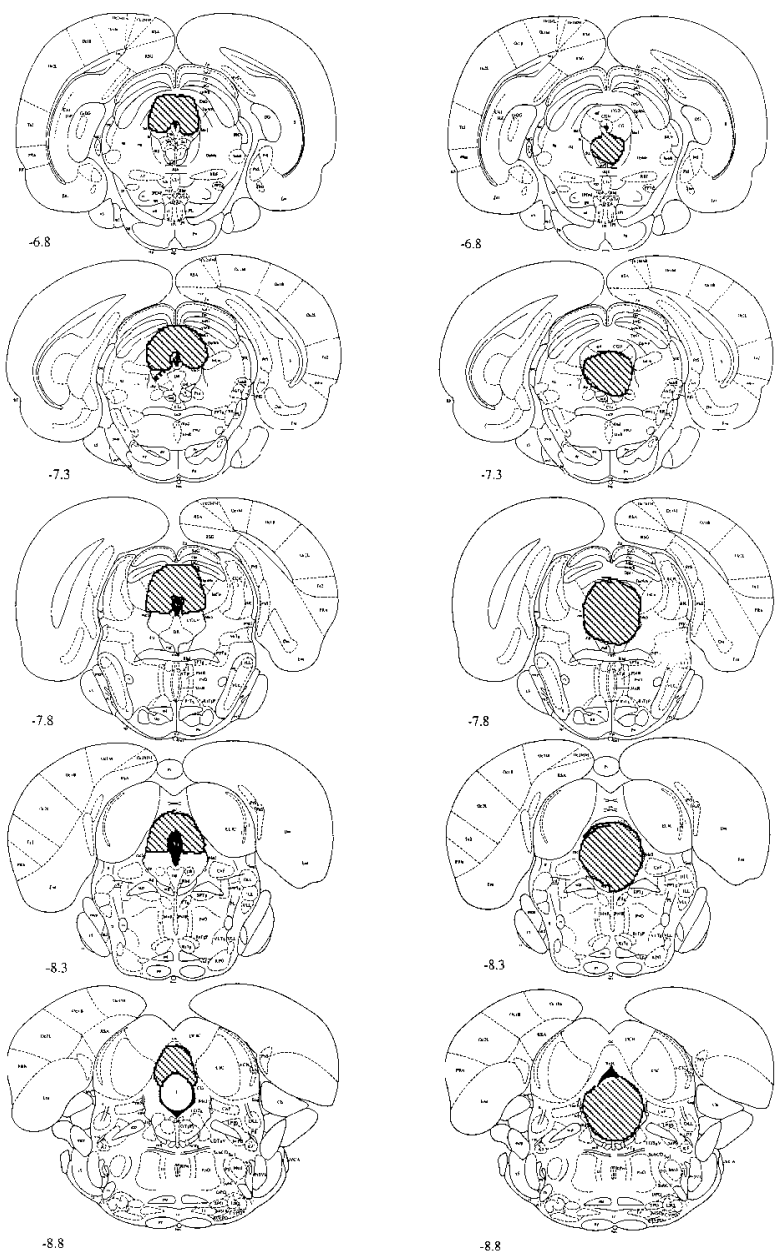

Figure 1. A representative sample of the location and extent of electrolytic lesions. Lesions of the dorsal PAG usually extended into the deep layers of the superior colliculus and the lateral PAG. Lesions of the ventral PAG included the lateral PAG and extended into the dorsal PAG.

peated two more times, providing a total of three measures of the intensities that elicited flinching, jumping, and vocalization.

In the fifth experiment, a $0.1 \%$ saccharin solution, novel to the rats, was used. A $0.15 \mathrm{M}$ intraperitoneal lithium chloride $(\mathrm{LiCl})$ injection was administered to induce toxicosis. All procedures were done in the home cages of the rats. For 5 d, rats were water deprived except for a daily 30 min period during which water was provided by placement of a water bottle on the cage of the rat. All bottles were placed inside the food hopper on the front of the cage. Food was thus removed from the food hoppers for each session and replaced after the $30 \mathrm{~min}$ session. At the end of each session, the amount of fluid consumed was recorded. On day 6 , all rats received a bottle containing a solution of $0.1 \%$ saccharin $(\mathrm{w} / \mathrm{v})$ instead of water for $30 \mathrm{~min}$. For sham-lesioned rats in the backwardpaired group, access to saccharin was preceded $2 \mathrm{hr}$ earlier by an intraperitoneal injection of $0.15 \mathrm{M} \mathrm{LiCl}$ at $2 \%$ body weight. This procedure does not support development of a conditioned taste aversion. For rats in the sham, dIPAG, and vPAG forward-paired groups, access to saccharin was followed immediately by an injection of $\mathrm{LiCl}$. On day 7, all rats again received water for $30 \mathrm{~min}$ to compensate for reduced fluid intake on the conditioning day. On days $8-9$, all rats were provided with saccharin again for $30 \mathrm{~min}$ a day to assess saccharin consumption.

\section{RESULTS}

\section{Experiment 1: freezing to a cat}

\section{Histology}

Figure 1 shows the location and extent of PAG lesions. Lesions of the dIPAG typically extended into the deep layers of the superior colliculus and the lateral PAG. Lesions of the vPAG included the lateral PAG and in many cases extended into the dorsal PAG. Because lesions of the vPAG profoundly attenuate freezing to fear-conditioned CSs even when the dIPAG is also damaged (Fanselow et al., 1995), lesions were classified as vPAG lesions if they included the entire extent of the caudal vPAG, regardless of damage to the dlPAG. Typically, damage to the dlPAG was seen in the majority of the cases. However, no behavioral differences have ever been observed on freezing between subjects with vPAG lesions that included the dIPAG and subjects the lesions of which did not. Lesions were classified dIPAG lesions if they included the entire extent of the caudal dIPAG but spared the vPAG. This lesion includes the region of the PAG above the middle of the aqueduct and targets the entire dorsomedial and the dorsolateral columns of the PAG and the lateral column of the PAG using the description by Bandler and Shipley (1994). This resulted in 10 subjects in the sham group, 14 subjects in the vPAG group, and 6 subjects in the dlPAG group.

\section{Behavior}

As shown in Figure 2, during the baseline (PRE-CAT) apparatus exposure period, the rats froze very little. In the presence of the cat, sham-lesioned rats froze somewhat more, and lesions of the vPAG strongly attenuated this effect, with rats in this group freezing $<5 \%$ of the time. In contrast, lesions of the dlPAG enhanced the level of freezing such that rats in this group froze approximately twice as much as sham-lesioned rats did. There were a main effect of lesion $\left[F_{(2,27)}=7.3 ; p<0.005\right]$ and a main effect of repeated measure $\left[F_{(1,27)}=19.3 ; p<0.001\right]$. There was also a significant lesion $\times$ repeated-measure interaction $\left[F_{(2,27)}=9.9 ; p<0.001\right]$. Fisher post hoc comparisons indicate that all three groups of rats differed significantly from each other in the level of freezing displayed on the cat test. Furthermore, the amount of freezing in the dlPAG-lesioned group increased significantly on the cat test. The amount of freezing in the sham-lesioned group increased modestly on the cat test, although this effect was only marginally significant $(p<1.0)$.

\section{Experiment 2: conditional fear and dIPAG lesions Histology}

The lesions destroyed the entire caudal extent of the dIPAG above the middle of the aqueduct. Lesions often extended into the deep layers of the superior colliculus. Any subjects with either unilateral lesions or lesions destroying the caudal vPAG were eliminated from the behavioral analysis. See Table 1 for the number of subjects remaining in each group.

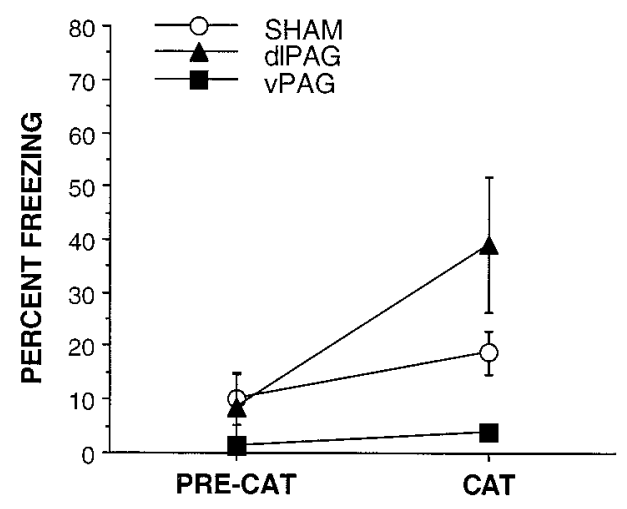

Figure 2. Rats with lesions of the dIPAG or the vPAG in comparison with sham-lesioned rats showed enhanced or decreased levels of freezing, respectively, when presented with a cat. 
Table 1. Number of subjects with dIPAG or sham pre- or post-training lesions in each shock condition

\begin{tabular}{llll} 
Day 3 & Days 11-13 & Day 16 & $n$ \\
\hline dlPAG lesion & Immediate shock & Sham lesion & 8 \\
dIPAG lesion & No shock & Sham lesion & 7 \\
Sham lesion & Immediate shock & dlPAG lesion & 6 \\
Sham lesion & No shock & dlPAG lesion & 4 \\
Sham lesion & Immediate shock & Sham lesion & 6 \\
Sham lesion & No shock & Sham lesion & 7 \\
\hline
\end{tabular}

\section{Behavior}

One subject from the sham-lesioned shock group was eliminated from the final analysis because it was an outlier; it froze $97 \%$ and was $>2$ SDs away from the group mean of $40.9 \%$ (including the outlier). In many experiments using this procedure, we have never seen a rat freeze at such a high level. Using this criterion, no other groups had an outlier. As shown in Figure 3, of the rats that received immediate shocks, only the rats that received lesions of the dlPAG before training showed levels of fear greater than that of the shocked controls. Freezing in the no-shock conditions varied from $\sim 12$ to $32 \%$. This is unusually high for animals that are not shocked and may reflect a small amount of conditioning or sensitization that could have occurred during the conditioning procedure. These animals were placed in the context just before the immediately shocked animals were placed in the context and shocked. Thus, the no-shock control animals experienced their placement in the context followed by the vocalizations of the immediately shocked rats. This may potentially account for the inflated freezing in the control groups. A one-way ANOVA revealed a significant main effect of group $\left[F_{(5,32)}=4.44 ; p<0.004\right]$. Fisher post hoc comparisons revealed a significant difference between the group receiving a pretraining dIPAG lesion with immediate shock and all other groups, which did not differ.

\section{Experiment 3: conditional fear and vPAG lesions Histology}

In general, the lesions damaged the ventral PAG bilaterally and extended into the dorsal raphé and into the ventrolateral PAG

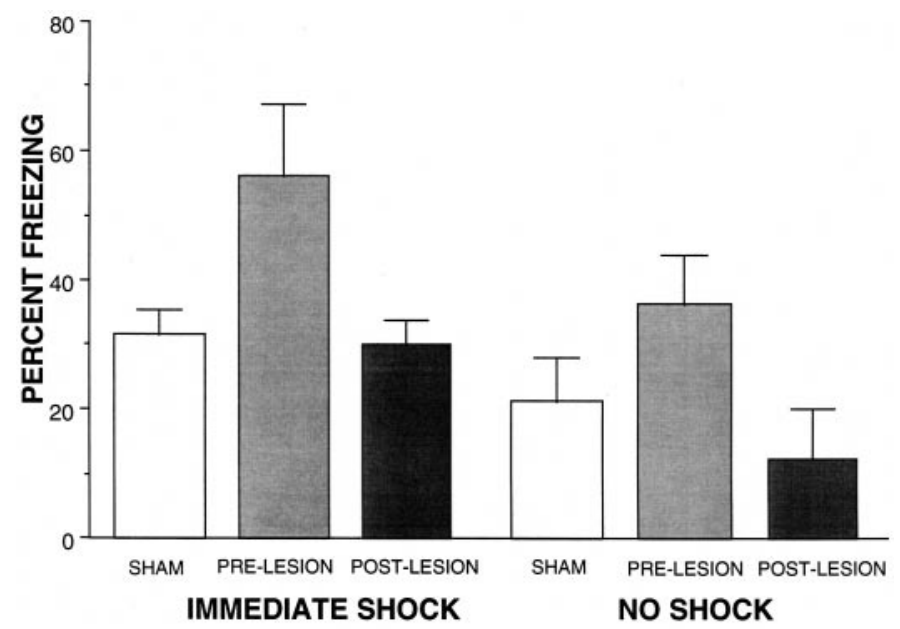

Figure 3. Sham-lesioned rats receiving an immediate shock after placement in a chamber freeze comparably with rats that were not shocked. Rats receiving a pretraining dIPAG lesion (PRE-LESION) showed enhanced levels of freezing after training with immediate shock, whereas rats receiving a post-training dIPAG lesion (POST-LESION) did not. Pre- and posttraining dIPAG lesions had no effect on rats that did not receive a shock.

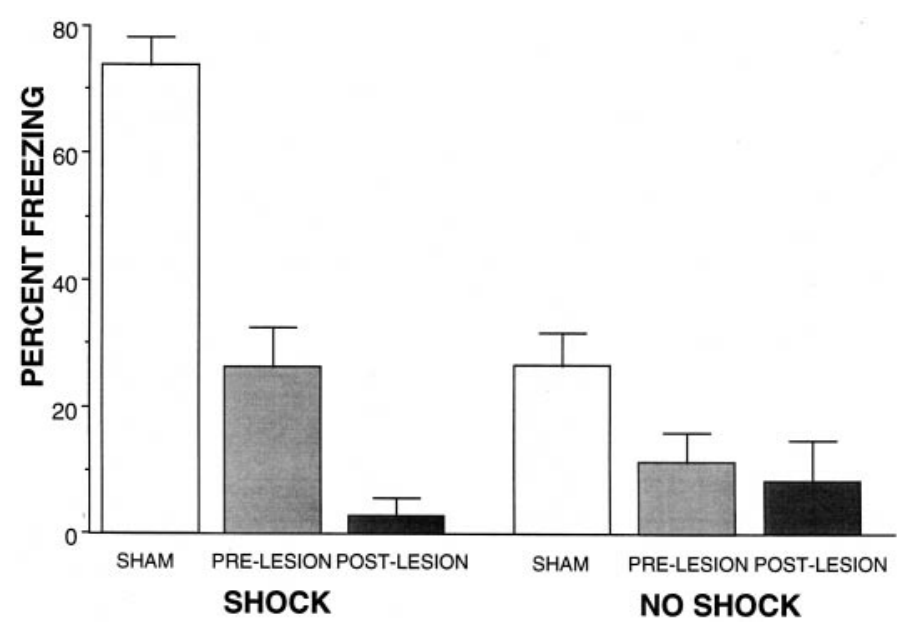

Figure 4. Rats receiving a vPAG lesion either before (PRE-LESION) or after (POST-LESION) training with shock showed reduced levels of freezing compared with the group receiving a sham lesion at both times. Furthermore, the group receiving a post-training lesion showed greater attenuation of freezing than did the group receiving a pretraining lesion.

and the lateral column of the PAG using the functional columns described by Bandler and Shipley (1994). Complete lesions of the caudal vPAG are required to attenuate freezing; incomplete lesions produce behavior comparable with that of sham lesions. Therefore, eight subjects were eliminated from the analysis because they had unilateral lesions or lesions that did not extend through the entire ventral PAG. This left eight subjects in both shock and no-shock sham-lesioned groups, six subjects each in the group receiving a lesion before training with shock and the group receiving a lesion before training with no shock, seven subjects in the group lesioned after training with shock, and five subjects in the group lesioned after training with no shock.

\section{Behavior}

As can be seen in Figure 4, only sham-lesioned rats that received shock during the training froze at a high level. Rats that received a vPAG lesion showed greatly reduced freezing behavior during the test. A one-way ANOVA detected a significant main effect of group $\left[F_{(5,34)}=23.165 ; p<0.0001\right]$. Fisher post hoc comparisons indicate that the groups of shocked rats receiving vPAG lesions before and after training were significantly different than the sham-lesioned controls. Additionally, lesions made after training with shock resulted in greater attenuation of freezing than did lesions made before training. Among the groups that did not receive shock, post-training lesions resulted in less freezing than did either sham or pretraining lesions.

\section{Experiment 4: flinch-jump-vocalization thresholds Behavior}

Rats with lesions of the vPAG or dIPAG did not differ from each other or from sham-lesioned rats in their latencies to flinch, jump, or vocalize. However, each of these behaviors was observed in response to increasing intensities of shock. Figure 5 illustrates these results. These observations were supported by the results of a repeated-measures ANOVA. There was no main effect of lesion condition $\left[F_{(2,27)}=1.2\right]$, but there was a significant main effect of response type $\left[F_{(2,27)}=197.9 ; p<0.0001\right]$. There was also no significant interaction between lesion condition and response type $\left[F_{(4,54)}<1.0\right]$. 


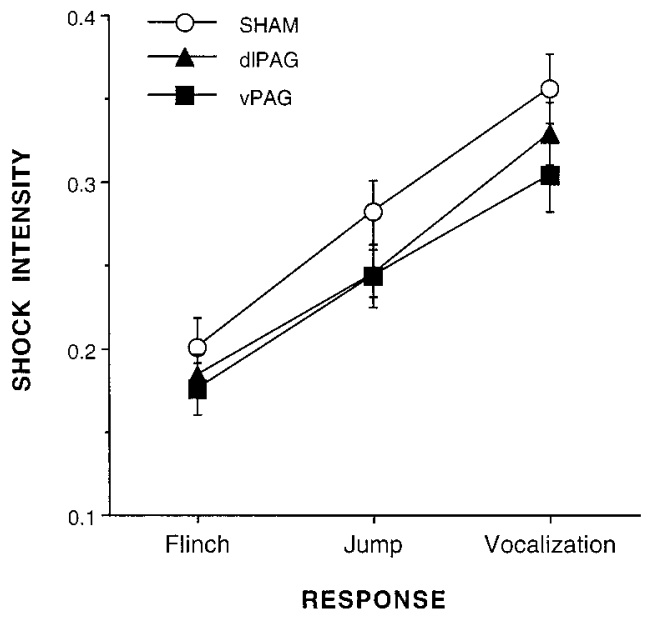

Figure 5. Rats with dIPAG, vPAG, or sham lesions did not differ in the shock intensity to which they flinched, jumped, or vocalized.

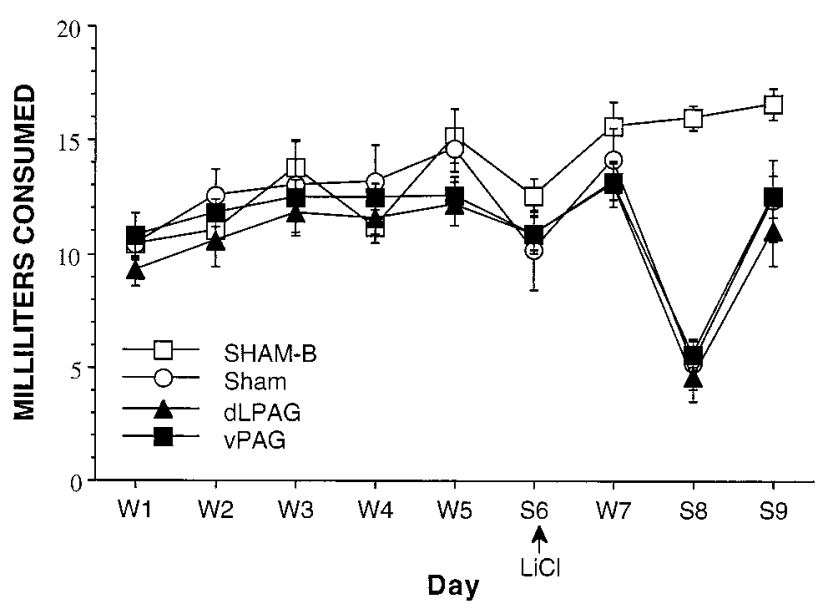

Figure 6. Rats with dlPAG, vPAG, or sham lesions did not differ in their consumption of a saccharin solution after toxicosis-induced taste aversion. $W$, Water; $S$, saccharin. Group SHAM-B received a backward pairing of saccharin and toxicosis and did not develop a taste aversion.

\section{Experiment 5: conditioned taste aversion}

All three groups receiving a forward pairing between saccharin and $\mathrm{LiCl}$ consumed reduced amounts of saccharin on day 8 regardless of the type of lesion made, indicating that equivalent levels of taste aversion developed in all three groups. Furthermore, all rats consumed less fluid on day 6, the first day that saccharin was provided. This reduced consumption is indicative of neophobia to the novel saccharin solution. Lesions of the PAG had no impact on this. A repeated-measures ANOVA confirmed these results. There were a main effect of group $\left[F_{(3,26)}=5.85\right.$; $p<0.005]$, a main effect of day $\left[F_{(2,52)}=148.32 ; p<0.0001\right]$, and a group $\times$ day interaction $\left[F_{(6,52)}=9.66 ; p<0.0001\right]$. Neither the dlPAG nor the vPAG lesions affected the acquisition or extinction of a conditioned taste aversion. Please see Figure 6 for a graphical representation of these results.

\section{DISCUSSION}

In the first experiment, we found that lesions of the vPAG and dIPAG produce different levels of cat-elicited freezing; vPAG lesions decreased freezing, whereas dIPAG lesions enhanced freezing. Freezing was not seen on the baseline day before cat exposure. Although the design confounds cat exposure with repeated exposure to the observation chamber, repeated exposure to a chamber alone does not elicit freezing (e.g., Young and Fanselow, 1992). Freezing is also distinct from sleeping or laying down that may occur when a rat is in a very familiar environment. The attenuation in freezing to the cat produced by vPAG lesions is consistent with the effect of these lesions on conditional freezing. The enhanced freezing to the cat produced by dlPAG lesions is not typically observed in standard conditioning paradigms. Although dlPAG lesions enhanced freezing, there is no other evidence that the mere presence of a danger stimulus produces dIPAG activity, because this area is typically involved in more active defensive responses to more imminent threats (Fanselow, 1991; Bandler and Shipley, 1994). This result is consistent with the view that dIPAG lesions may be removing tonic inhibition of the vPAG by the dIPAG (Fanselow, 1991).

Also, the enhanced freezing in rats with dIPAG lesions is consistent with the view that the dlPAG inhibits the amygdala. Removal of this inhibitory input may allow greater activity in the efferents of the amygdala, including the vPAG. However, research conducted in the laboratory of Helmstetter suggests otherwise. Lesions of the vPAG and the dlPAG blocked fear-conditioned hypoalgesia but not the arterial blood pressure response to the CS (Helmstetter and Tershner, 1994). If the dlPAG inhibited the amygdala, lesions of the dIPAG should, if anything, have facilitated the hypoalgesia and the arterial blood pressure response seen in that experiment. Because lesions of the dlPAG do not block unconditional hypoalgesia (Bellgowan and Helmstetter, 1996), it cannot be necessary for the hypoalgesic response.

Another claim that can be made is that some conditioning occurs to the context with the cat as a US. dIPAG lesions may potentially enhance this type of normally weak conditioning. However, this is not likely to be a major contributor to the behavior of the rats in this experiment because of the short duration of the test and the low levels of fear behaviors produced by the cat. In sum, it is more likely that the enhanced freezing seen here is supportive of the proposed tonic inhibition of Fanselow (1991) of the vPAG by the dlPAG. Lesions of the dlPAG may enhance freezing to weak stimuli like the cat by removal of this intra-PAG tonic inhibition.

The hypothesis that the dPAG is involved during acquisition was tested in experiment 2. Lesions of the dlPAG are known to attenuate the immediate shock deficit (Fanselow et al., 1995). In experiment 2 , this effect was observed only when lesions were made before but not after training. Thus, the dlPAG exerts an inhibitory effect during acquisition with massed and immediate shock but has no critical role during performance. The immediate shock deficit is a deficit in acquisition and not in performance (Fanselow, 1986). Thus, it is the result of a deficit in either CS or US processing. Because dlPAG lesions do not enhance US sensitivity (experiment 4), the deficit seems to be one of CS processing and probably involves the amygdala (Maren and Fanselow, 1996) or other forebrain areas. The tonic inhibition of the vPAG by the dIPAG proposed earlier by Fanselow (1991) cannot readily explain this result because stimulation of the vPAG elicits freezing but not the autonomic responses to fear-eliciting stimuli (Lovick, 1991). If the vPAG stimulated the amygdala, that should produce all of the components of the defensive response because amygdala stimulation elicits all of the components of the defensive response (Applegate et al., 1983; Ohta et al., 1991). Thus, the most likely possibility is that the dPAG may briefly inhibit the amygdala or other forebrain structures and interfere with the 
processing of incoming sensory information, thereby preventing processing of the CS during footshock administration.

Neuroanatomical studies have demonstrated that circuitry that could mediate such a dIPAG-amygdala inhibitory connection exists. First, the amygdala receives afferents from the dlPAG. Although the majority of these projections originate in the rostral PAG, there are some PAG fibers that project to the amygdala in the caudal dlPAG (Rizvi et al., 1991). The caudal dIPAG lesions made in the reported experiments may either disrupt intra-PAG communication necessary for this inhibitory signal to the amygdala or damage a sufficient number of fibers to disrupt the inhibition of the amygdala. Furthermore, throughout the rostral-caudal extent of the PAG, fibers originating in the PAG innervate various forebrain areas through the medial forebrain bundle (Cameron et al., 1995). In summary, the anatomical connections between the PAG and the amygdala and other forebrain structures exist that could mediate the kind of inhibition proposed by Fanselow (1994).

There are other data that indicate the functional relevance of this kind of inhibitory circuit as well. Circastrike attack is not one of the responses elicited by direct stimulation of the central nucleus of the amygdala. Indeed, stimulation of the lateral amygdala and central nucleus of the amygdala produces long-lasting, opioidmediated inhibition of the affective defensive response elicited by dlPAG stimulation in the cat (Shaikh et al., 1991). Importantly, this inhibition was selective to defensive behavior, because circling behavior elicited by dIPAG stimulation in one cat was unaffected by amygdala stimulation. Thus, it may be necessary for the amygdala to be inhibited in order to engage in active defensive behaviors like circastrike attack. It may be that in times of physical contact between predator and prey, the defensive needs of the animal are best served by complete midbrain control and activation of circastrike behaviors. Once the organism is no longer under direct physical attack, forebrain activity mediating freezing reduces the attractiveness of the prey to the predator (Sargeant and Eberhardt, 1975; Thompson et al., 1981). Also, forebrain activity is necessary to monitor the environment and to allow for retreat to a safer area and eventual return to the preferred activity pattern once the predator has departed the immediate area (Fanselow and Lester, 1988).

The results obtained here suggest that the dIPAG inhibits acquisition of conditional fear. However, Walker and Davis (1997) eliminated the attenuation of fear-potentiated startle seen in the presence of a cue fear-conditioned with high footshock intensities using post-training lesions. Walker and Davis correctly conclude that the role of the dIPAG in potentiated startle seems to be during performance of fear-motivated behaviors and not during acquisition. They suggest that the dlPAG inhibits presynaptic amygdalar inputs to the nucleus reticularis pontis caudalis, a critical part of the fear-potentiated startle circuit. Walker and Davis (1997) also point out that other behaviors like defecation do not decrease when elicited by cues trained with high shock intensities. This would not be possible if the dIPAG inhibits the expression of fear during testing.

Considering the lack of an effect on defecation elicited by cues fear-conditioned with high shock intensities and the effect of posttraining lesions on fear-potentiated startle, it seems that the results reported here are inconsistent with those provided by Walker and Davis (1997). However, the results of both sets of studies are consistent if what is considered is the participation of the dIPAG during extreme fear. Ratner (1967) proposed a description of defensive response topography that varied as a function of the distance between predator and prey. Defensive behaviors varied between freezing, flight, fight, and tonic immobility as the predatory distance decreased. Tonic immobility is a prone, immobile position elicited in wild or naive rats and in lizards as well as in chickens and other prey animals. It is thought to inhibit further attack by removing movement as an attack-eliciting cue (Sargeant and Eberhardt, 1975). Fear levels would theoretically be inversely related to the predatory distance (Fanselow and Lester, 1988). Thus, if dIPAG activity is elicited by extreme levels of fear, there may be a tendency to inhibit "active" behavioral defenses and facilitate "passive" behavioral defenses according to the response topography of Ratner (1967), at least until any contact by the predator causes the rat to engage in circastrike defensive attack. Thus, dIPAG lesions may have the effect of attenuating fearpotentiated startle via the neuroanatomical connections proposed by Walker and Davis (1997), because potentiated startle is incompatible with tonic immobility but may have no such effect on the performance of conditional freezing.

In contrast to the role in performance behaviors elicited by extremely high levels of fear, the role of dIPAG in acquisition of moderate fear is more general. Shocks during acquisition of conditional fear may briefly interfere with the associative process, and this interference may be mediated by the dIPAG. The deficit seen with massed or immediate shock delivery can only be accounted for by a deficit in learning (Fanselow et al., 1993; Fanselow, 1986). Both freezing deficits are accompanied by deficits in conditional analgesia, and the immediate shock deficit is accompanied by a deficit in fear-elicited defecation (Fanselow, 1986). Thus, lesions of the dIPAG probably eliminate the massed and immediate shock deficits by removing this brief inhibition of associative processes occurring outside of the midbrain. The exact location of these inhibitory inputs is unknown. Although the amygdala is a good candidate, inactivation of the basolateral amygdala with GABA agonists during acquisition results in only a small attenuation of conditional fear compared with inactivation of this area during performance (Helmstetter and Bellgowan, 1994).

In contrast with the demonstrated role of the dlPAG in the acquisition of fear conditioning, the vPAG was shown to be necessary for the performance of conditional freezing. There is no ready explanation for the greater attenuation of freezing when the lesions were made after training, but both pre- and post-training lesions produced levels of freezing equivalent or less to that of the no-shock control group. The attenuation in freezing in the experimental groups cannot be attributed to a deficit in US processing because post-training lesions were sufficient to attenuate conditional freezing. Furthermore, vPAG lesions had no effect on footshock sensitivity (experiment 4 ). Finally, the ability of vPAG lesions to reduce freezing to an innate danger stimulus, a cat, also supports the role of this structure in the expression of freezing.

This finding is entirely consistent with the neuroanatomical connections within the PAG and between the amygdala and the vPAG. Stimulation of the amygdala produces freezing along with the cardiovascular and respiratory changes that accompany the behavioral defensive response (Applegate et al., 1983; Ohta et al., 1991). These are the same responses produced by stimulation of the lateral column of the PAG (Depaulis et al., 1994), a region of the caudal PAG included in the lesions made here of the vPAG. Because this region is included in both the dIPAG and the vPAG lesions of the experiments reported here, the differential effects seen in experiments 1-3 cannot be attributed to this area but may be attributed to the more dorsal aspect of the dIPAG lesion and the more ventral aspect of the vPAG lesion. Also, lesions studies indicate that the amygdala and the PAG do not have identical 
functions. Amygdala central nucleus lesions block all conditional fear responses including behavioral, autonomic, cardiovascular, and hormonal responses, but lesions of the PAG block only the behavioral responses to fear (LeDoux, 1996). Thus, it is believed that the central nucleus of the amygdala may be the final common pathway of conditional fear responses and that its efferent targets, including the PAG, mediate specific responses. In support of this view, the $\mathrm{GABA}_{\mathrm{A}}$ agonist muscimol significantly attenuated conditional freezing when applied to the amygdala before acquisition and before testing (Helmstetter and Bellgowan, 1994). As mentioned previously, the effect was greatest at the time of testing, suggesting a critical role for amygdala $\mathrm{GABA}_{\mathrm{A}}$ neurons during the expression of conditional fear. These results also suggest that the vPAG may also be under tonic GABA inhibition that is removed during the expression of conditional fear.

Furthermore, the central nucleus of the amygdala contains enkephalinergic efferents to the dlPAG. These inhibitory connections are fast in comparison to the excitatory efferents to the vPAG, and they seem to act on intra-PAG inhibitory connections that because of their high level of baseline activity appear to be tonically active (Da Costa Gomez and Behbehani, 1995). This scenario is consistent with that proposed by Fanselow (1991), whereby the dlPAG tonically inhibits the freezing response of the vPAG. Indeed, dlPAG stimulation does inhibit vPAG neurons (Chandler et al., 1993; as cited in Behbehani, 1995).

In summary, different regions of the PAG seem to play distinct roles in defensive behavior. Overall, the role of the vPAG emerging from this and previous work is one of a structure that critically mediates freezing to stimuli that engage the postencounter stage of defense of the animal. In contrast, the role of the dlPAG emerging from this work is that of a structure that can inhibit activity in forebrain structures during times of extreme risk, such as that elicited by shock and predatory attack.

\section{REFERENCES}

Applegate CD, Kapp BS, Underwood MD, McNall CL (1983) Autonomic and somatomotor effects of amygdala central nucleus stimulation in awake rabbits. Physiol Behav 31:353-360.

Bandler R, Shipley MT (1994) Columnar organization in the midbrain periaqueductal gray: modules for emotional expression. Trends Neurosci 17:379-389.

Behbehani MM (1995) Functional characteristics of the midbrain periaqueductal gray. Prog Neurobiol 46:575-605.

Bellgowan PS, Helmstetter FJ (1996) Neural systems for the expression of hypoalgesia during nonassociative fear. Behav Neurosci 110:727-736.

Blanchard RJ, Blanchard DC (1969) Crouching as an index of fear. J Comp Physiol Psychol 67:370-375.

Blanchard RJ, Blanchard DC (1971) Defensive reactions in the albino rat. Learn Motiv 2:351-362.

Bolles RC, Collier AC (1976) The effect of predictive cues on freezing in rats. Anim Learn Behav 4:6-8.

Borszcz GS, Cranney J, Leaton RN (1989) Influence of long-term sensitization on long-term habituation of the acoustic startle response in rats: central gray lesions, preexposure, and extinction. J Exp Psychol [Anim Behav Proc] 15:54-64.

Cameron AA, Khan IA, Westlund KN, Cliffer KD, Willis WD (1995) The efferent projections of the periaqueductal gray in the rat: a Phaseolus vulgaris-leucoagglutinin study. I. Ascending projections. J Comp Neurol 351:568-584.

Da Costa Gomez TM, Behbehani MM (1995) An electrophysiological characterization of the projection from the central nucleus of the amygdala to the periaqueductal gray of the rat: the role of opioid receptors. Brain Res 689:21-31.

Davis M, Astrachan DI (1978) Conditioned fear and startle magnitude: effects of different footshock or backshock intensities used in training. J Exp Psychol [Anim Behav Proc] 4:95-103.

Depaulis A, Keay KA, Bandler R (1994) Quiescence and hyporeactivity evoked by activation of cell bodies in the ventrolateral midbrain periaqueductal gray of the rat. Exp Brain Res 99:75-83.

Fanselow MS (1980) Conditioned and unconditional components of post-shock freezing. Pavlov J Biol Sci 15:177-182.

Fanselow MS (1986) Associative vs topographical accounts of the immediate shock-freezing deficit in rats: implications for the response selection rules governing species-specific defensive reactions. Learn Motiv $17: 16-39$.

Fanselow MS (1991) The midbrain periaqueductal gray as a coordinator of action in response to fear and anxiety. In: The midbrain periaqueductal gray matter. Functional, anatomical, and neurochemical organization (Depaulis A, Bandler R, eds). New York: Plenum.

Fanselow MS (1994) Neural organization of the defensive behavior system responsible for fear. Psychonomic Bull Rev 1:429-438.

Fanselow MS, Lester LS (1988) A functional behavioristic approach to aversively motivated behavior: predatory imminence as a determinant of the topography of defensive behavior. In: Evolution and learning (Bolles RC, Beecher MD, eds). Hillsdale, NJ: Erlbaum.

Fanselow MS, Tighe TJ (1988) Contextual conditioning with massed versus distributed unconditional stimuli in the absence of explicit conditional stimuli. J Exp Psychol [Anim Behav Proc] 14:187-199.

Fanselow MS, DeCola JP, Young SL (1993) Mechanisms responsible for reduced contextual conditioning with massed unsignaled unconditional stimuli. J Exp Psychol [Anim Behav Proc] 19:121-137.

Fanselow MS, DeCola JP, De Oca BM, Landeira-Fernandez J (1995) Ventral and dorsolateral regions of the midbrain periaqueductal gray (PAG) control different stages of defensive behavior: dorsolateral PAG lesions enhance the defensive freezing produced by massed and immediate shock. Aggressive Behav 21:63-77.

Helmstetter FJ, Bellgowan PS (1994) Effects of muscimol applied to the basolateral amygdala on acquisition and expression of contextual fear conditioning in rats. Behav Neurosci 108:1005-1009.

Helmstetter FJ, Tershner SA (1994) Lesions of the periaqueductal gray and rostral ventromedial medulla disrupt antinociceptive but not cardiovascular aversive conditional responses. J Neurosci 14:7099-7108.

Leaton RN, Borszcz GS (1985) Potentiated startle: its relation to freezing and shock intensity in rats. J Exp Psychol [Anim Behav Proc] 11:421-428.

LeDoux JE (1996) Emotional networks and motor control: a fearful view. In: Progress in brain research, Vol 107 (Holstege G, Bandler R, Saper CB, eds), pp 437-446.

LeDoux JE, Iwata J, Cicchetti P, Reis DJ (1988) Different projections of the central amygdaloid nucleus mediate autonomic and behavioral correlates of conditioned fear. J Neurosci 8:2517-2529.

Lovick TA (1991) Interactions between descending pathways from the dorsal and ventrolateral periaqueductal gray matter in the rat. In: The midbrain periaqueductal gray matter. Functional, anatomical, and neurochemical organization (Depaulis A, Bandler R, eds). New York: Plenum.

Maren S, Fanselow MS (1996) The amygdala and fear conditioning: has the nut been cracked? Neuron 16:237-240.

Ohta H, Watanabe S, Ueki S (1991) Cardiovascular changes induced by chemical stimulation of the amygdala in rats. Brain Res Bull 26:575-581.

Ratner SC (1967) Comparative aspects of hypnosis. In: Handbook of clinical and experimental hypnosis (Gordon JE, ed). New York: Macmillan.

Rizvi TA, Ennis M, Behbehani MM, Shipley MT (1991) Connections between the central nucleus of the amygdala and the midbrain periaqueductal gray: topography and reciprocity. J Comp Neurol 303:121-131.

Sargeant AB, Eberhardt LE (1975) Death feigning by ducks in response to predation by red foxes (Vulpes fulva). Midland Naturalist 93:108-119.

Shaikh MB, Lu CL, Siegel A (1991) An enkephalinergic mechanism involved in amygdaloid suppression of affective defense behavior elicited from the midbrain periaqueductal gray in the cat. Brain Res 559:109-117.

Thompson RKR, Foltin RW, Boylan RJ, Sweet A, Graves CA, Lowitz CE (1981) Tonic immobility in Japanese quail can reduce the probability of sustained attack by cats. Anim Learn Behav 9:145-149.

Walker DL, Davis M (1997) Involvement of the dorsal periaqueductal gray in the loss of fear-potentiated startle accompanying high footshock training. Behav Neurosci 111:692-702.

Young SL, Fanselow MS (1992) Associative regulation of Pavlovian fear conditioning: unconditional stimulus intensity, incentive shifts and latent inhibition. J Exp Psychol [Anim Behav Proc] 18:400-413. 